\title{
An Adaptive Image Denoising Model Based on Tikhonov and TV Regularizations
}

\author{
Kui Liu, ${ }^{1,2}$ Jieqing Tan, ${ }^{1}$ and Benyue $\mathrm{Su}^{2}$ \\ ${ }^{1}$ School of Computer and Information, Hefei University of Technology, Hefei 23009, China \\ ${ }^{2}$ School of Computer and Information, Anqing Normal University, Anqing 246011, China \\ Correspondence should be addressed to Kui Liu; liukui@aqtc.edu.cn
}

Received 23 January 2014; Revised 11 July 2014; Accepted 14 July 2014; Published 4 August 2014

Academic Editor: Jianping Fan

Copyright (c) 2014 Kui Liu et al. This is an open access article distributed under the Creative Commons Attribution License, which permits unrestricted use, distribution, and reproduction in any medium, provided the original work is properly cited.

\begin{abstract}
To avoid the staircase artifacts, an adaptive image denoising model is proposed by the weighted combination of Tikhonov regularization and total variation regularization. In our model, Tikhonov regularization and total variation regularization can be adaptively selected based on the gradient information of the image. When the pixels belong to the smooth regions, Tikhonov regularization is adopted, which can eliminate the staircase artifacts. When the pixels locate at the edges, total variation regularization is selected, which can preserve the edges. We employ the split Bregman method to solve our model. Experimental results demonstrate that our model can obtain better performance than those of other models.
\end{abstract}

\section{Introduction}

Images are inevitably contaminated by noise during acquisition and transmission and so image denoising is one of the most fundamental tasks in image processing and computer vision [1]. Image denoising aims at preserving edges and finescale structural information while removing the noise in the image. During the past two decades, a great deal of research [2-4] has gone into excogitating models for removing noise while preserving edges and other fine-scale image details. Recently, partial differential equation (PDE) and variationalbased methods have gained popularity for image denoising $[5,6]$. Among them, the best known one is TV model proposed by Rudin et al. [7]. Although this second-order PDE can succeed in preserving edges, it suffers from the staircase effect in the smooth regions. To prevent the staircasing, a modified model proposed by Blomgren et al. [8] was to automatically adapt the gradient-based exponent to fit the data term. It has the following form:

$$
\min _{u \in \operatorname{BV}(\Omega)}\left\{\iint_{\Omega}\left(|\nabla u|^{p(|\nabla u|)}+\frac{\lambda}{2}\left(u-u_{0}\right)^{2}\right) d x d y\right\} .
$$

Gradient-based exponent has value 1 near the edges and is close to 2 in the smooth region, so that near the edges it behaves exactly like the ROF model and behaves like Tikhonov regularization method in the smooth regions. However, this model is nonconvex and difficult to solve. Another approach to overcome the limitation of TV is to introduce higher-order derivatives into the energy function $[9,10]$. One of the most classical fourth-order PDEs is introduced by You and Kaveh (Y-K), which measured the oscillations in the noisy image according to the two different functions [11]. But, a major challenge higher-order PDEs face is numerical computation. At the same time, another challenge is to blur the edges in the denoising. In addition, they can also produce speckle noise in the recovered image. Recently, some hybrid models were proposed which combined the second-order partial differential equation models and the fourth-order PDEs [12, 13]. Gholami and Hosseini [14] proposed a novel method to combine total variation regularization and Tikhonov regularization to reconstruct piecewise smooth signals.

Inspired by their work, we propose an adaptive image denoising model by the weighted combination of Tikhonov regularization and total variation regularization. Unlike the model [14] which firstly divided the image into piecewise constant component and smooth component, our model directly uses the gradient information to define the weight 
functions which judge whether the pixels locate at the edges or the smooth regions. When the pixels belong to the smooth regions, Tikhonov regularization is adopted, which can eliminate the staircase artifacts. When the pixels locate at the edges, total variation regularization is selected, which can preserve the edges. Our model does not solve the highorder PDE and is also a convex combination. All numerical experiments verify the efficiency of our proposed model.

The remainder of this paper is organized as follows. In Section 2, we briefly review Tikhonov regularization and TV regularization and then discuss their advantages and limitations. In Section 3, we give the proposed model and numerical implementation in detail. The experimental results are given in Section 4. Finally, this paper is concluded in Section 5 .

\section{Related Works and Discussion}

In this paper, the observed noisy image $u_{0}\left(\Omega \subset R^{2} \rightarrow R\right)$ can be defined as $u_{0}=u+n$; here, $u$ is the original image, and $n$ is an additive Gaussian white noise with the mean 0 and variance $\sigma^{2}$. Mathematically speaking, the denoising problem is an ill-posed inverse problem. One of the most known techniques to solve this problem is regularization method and energy minimization as follows:

$$
u=\min _{u}\left\{R(u)+\frac{\lambda}{2}\left(u-u_{0}\right)^{2}\right\} .
$$

Here, the first term $R(u)$ is a regularization term, while the second term is fidelity term which ensures that the denoised image is close to the observed image, and $\lambda>0$ is the regularization parameter which balances the regularization term and the fidelity one. Much research has been put forward to remove the noises from the noisy image by either exploiting a particular regularization technique $[15,16]$ or taking the advantages of a set of regularizations using a combination of them $[14,17,18]$. In the image denoising, Tikhonov regularization and total variation regularization are two kinds of regularization techniques and they are widely used in the past three decades.

2.1. Tikhonov Regularization. Tikhonov regularization method is one of the earliest used regularizations, in which the cost function contains an $L_{2}$-norm regularization term of the magnitude of the image gradient [19]. The model based on the Tikhonov regularization method is defined as follows:

$$
\min _{u}\left\{\iint_{\Omega}\left(\|\nabla u\|_{2}^{2}+\frac{\lambda}{2}\left(u-u_{0}\right)^{2}\right) d x d y\right\},
$$

where $\|\cdot\|_{2}$ is $L_{2}$ norm of the bounded variation.

We know that minimizing formula (3) yields the EulerLagrange equation

$$
u_{t}=\Delta u+\lambda\left(u_{0}-u\right)
$$

Seen from (4), Tikhonov regularization technique is actually isotropic diffusion. Hence, while removing the noise in the observed image $u_{0}$, prominent structures like edges and jumps in the denoised image $u$ are blurred. However, the first- and second-order derivative operators are only used in Tikhonov regularization, so it has computational advantages [20].

2.2. Total Variation Regularization. In addition, there are other regularization techniques which preserve the edge information in the recovered image [21]. Among these edgepreserving regularization methods [7], the best known one is the total variation regularization. Rudin et al. proposed using the $L_{1}$ norm of the gradient of the image, instead of the $L_{2}$ norm, so the formulation for the variational model (ROF) is as follows:

$$
\min _{u}\left\{\iint_{\Omega}\left(\|\nabla u\|_{\mathrm{TV}}+\frac{\lambda}{2}\left(u-u_{0}\right)^{2}\right) d x d y\right\},
$$

subject to

$$
\begin{aligned}
& \iint_{\Omega} u(x, y) d x d y=\iint_{\Omega} u_{0}(x, y) d x d y \\
& \frac{1}{|\Omega|} \iint_{\Omega}\left(u(x, y)-u_{0}(x, y)\right)^{2} d x d y=\sigma^{2}
\end{aligned}
$$

Here $\Omega$ is a bounded domain of the image with Lipschitz boundary. $\operatorname{BV}(\Omega)$ is the space of functions with bounded variation. $|\Omega|$ represents the area of the image. By the gradient descent method, we get the Euler-Lagrange equation of TV denoising model from (5) as follows:

$$
\begin{aligned}
& u_{t}=\nabla \cdot\left(\frac{\nabla u}{|\nabla u|}\right)+\lambda\left(u_{0}-u\right), \\
& \left.u(x, y, t)\right|_{t=0}=u_{0}(x, y) .
\end{aligned}
$$

When $|\nabla u|=\sqrt{\left|\nabla_{x} u\right|^{2}+\left|\nabla_{y} u\right|^{2}}$, formula (5) is the isotropic TV of $u$. $\nabla_{x} u$ and $\nabla_{y} u$ are the horizontal and the vertical gradient operators, respectively. The isotropic TV regularization performs well in preserving edges while eliminating noise. However, the fine-scale details such as textures are often smoothed out with noise in the image. Esedoglu and Osher [21] proposed anisotropic total variation (ATV) regularization as $|\nabla u|=\left|\nabla_{x} u\right|+\left|\nabla_{y} u\right|$. The uniqueness and existence of solutions of ATV are shown in [22]. However, the ATV is still TV-type regularization which is not good at preserving fine-scale details and may cause staircase artifacts in the smooth regions.

\section{The Proposed Method}

3.1. The New Model. Generally, the natural images contain the smooth regions with sharp edges. According to what is mentioned above, the TV-type regularization can effectively remove noise and preserve sharp edges, but it easily causes staircase effects in the flat regions. On the other hand, Tikhonov regularization can eliminate the staircase effect although it blurs the edges. Therefore, in order to eliminate staircase effect and preserve fine-scale details, we combine 


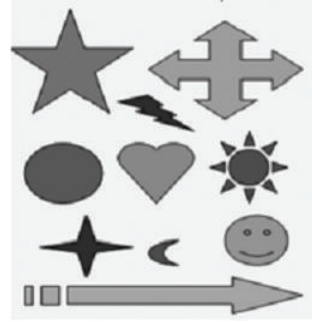

(a)

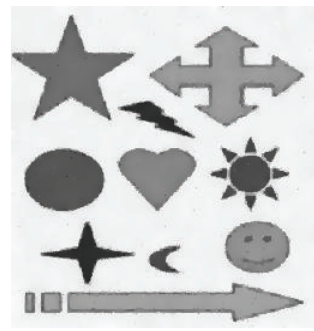

(e)

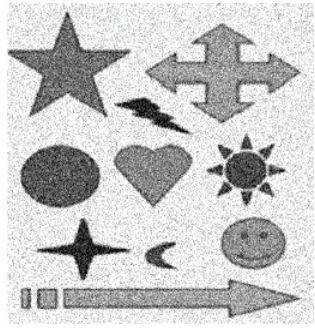

(b)

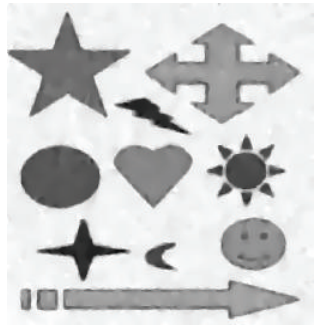

(f)

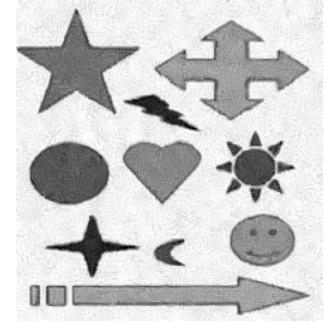

(c)

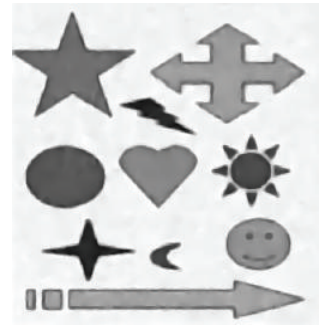

(g)

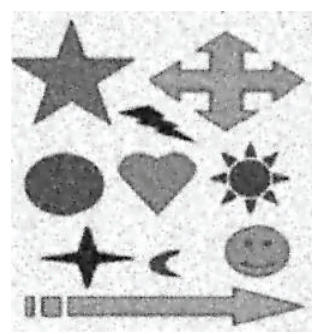

(d)

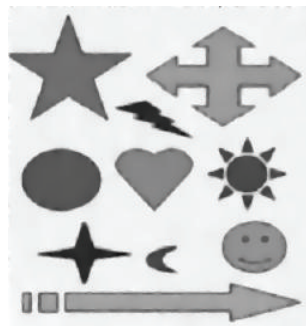

(h)

FIGURE 1: Result of the synthetic image by the five models: (a) original image, (b) noisy image, (c) result by NLM, (d) result by Y-K model, (e) result by PM, (f) result by TV, (g) result by MTV, and (h) result by our model.

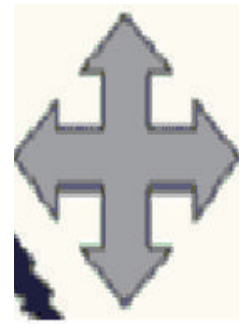

(a)

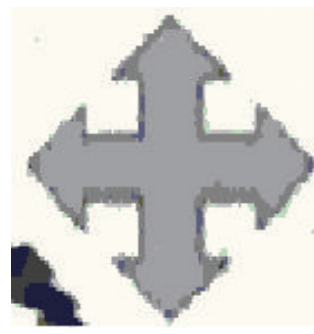

(e)

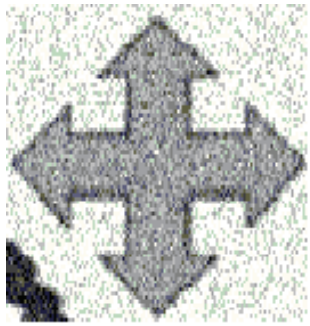

(b)

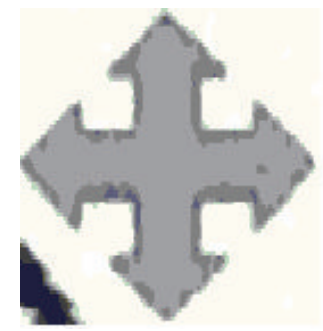

(f)

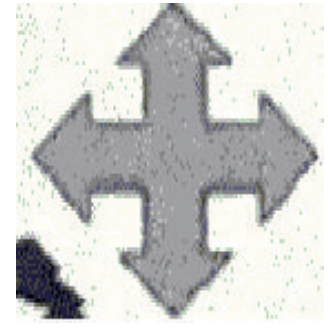

(c)

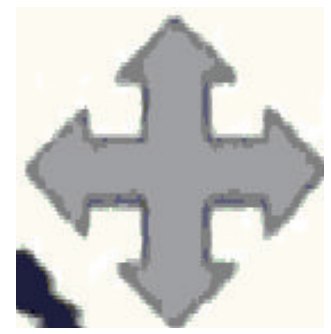

(g)

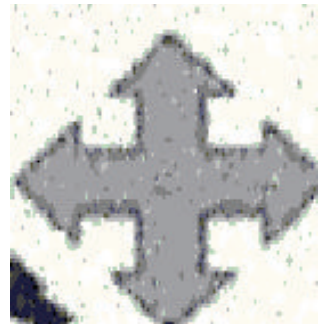

(d)

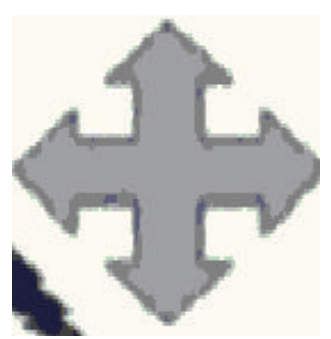

(h)

FIGURE 2: A part of the results of the synthetic image by the five models: (a) original image, (b) noisy image, (c) result by NLM, (d) result by Y-K model, (e) result by PM, (f) result by TV, (g) result by MTV, and (h) result by our model.

these two regularizations and take the energy function of the image for image denoising as follows:

$$
\begin{gathered}
\min _{u}\left\{\int \int _ { \Omega } \left[(1-\varphi(s))\|\nabla u\|_{\mathrm{TV}}+\varphi(s)\|\nabla u\|_{2}^{2}\right.\right. \\
\left.\left.+\frac{\lambda}{2}\left(u-u_{0}\right)^{2}\right] d x d y\right\} .
\end{gathered}
$$

Here the functions $\varphi(s)$ are defined as

$$
\varphi(s)=\exp \left(-\left(\frac{s}{K}\right)^{2}\right)
$$

where $K$ is constant and $s$ is defined as the image gradient modulus value. The function $\varphi(s) \in[0,1]$ decreases monotonically from 1 to 0 . When the pixels belong to edges, the value of $s$ is large, $\operatorname{so} \varphi(s) \rightarrow 1$. When the pixels belong to flat 


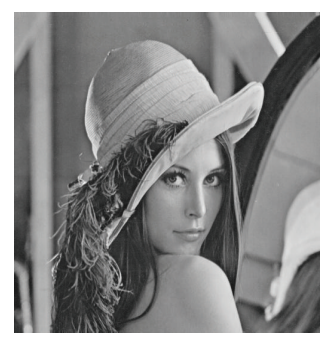

(a)

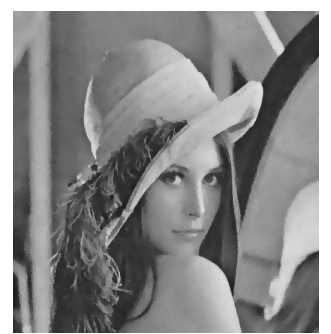

(e)

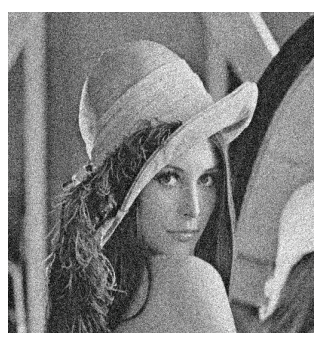

(b)

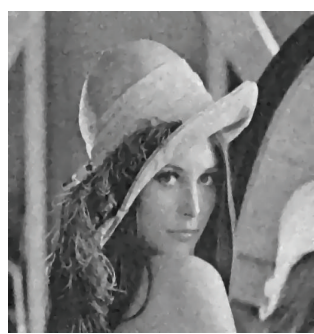

(f)

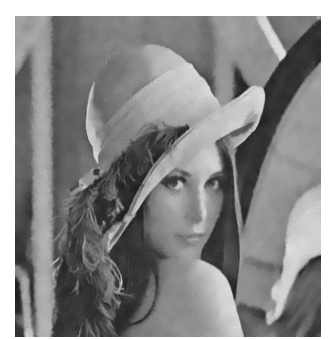

(c)

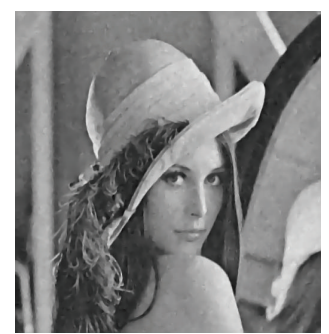

(g)

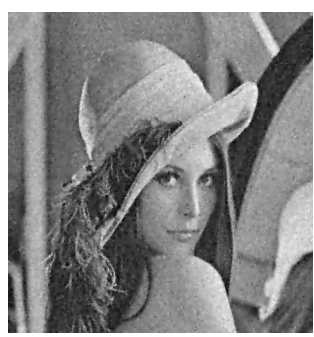

(d)

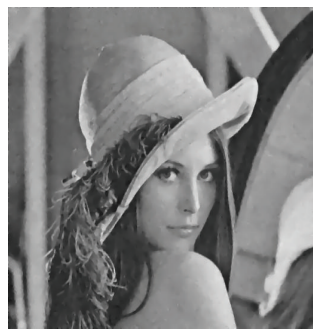

(h)

FIGURE 3: Results of the lena image by the five models: (a) original image, (b) noisy image, (c) result by NLM, (d) result by Y-K model, (e) result by PM, (f) result by TV, (g) result by MTV, and (h) result by our model.

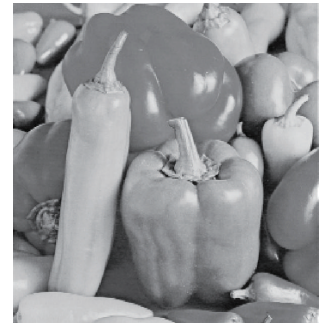

(a)

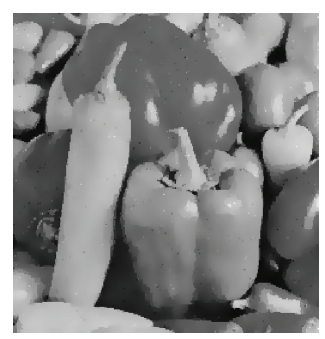

(e)

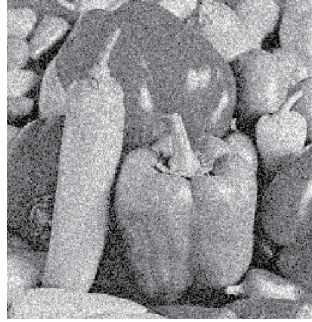

(b)

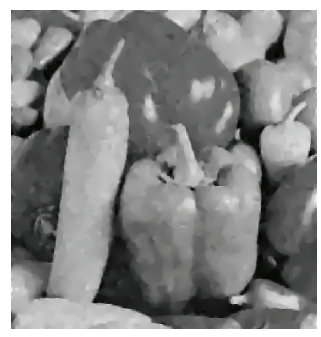

(f)

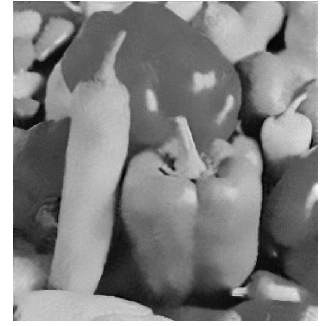

(c)

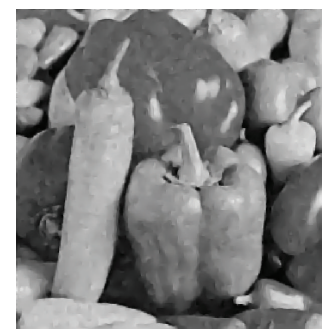

(g)

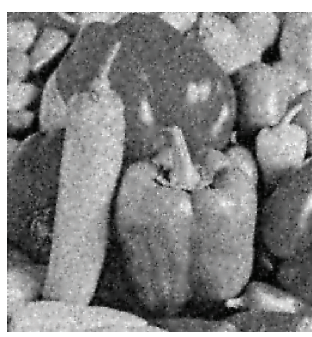

(d)

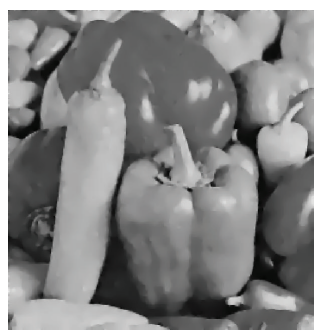

(h)

FIGURE 4: Results of the peppers image by the five models: (a) original image, (b) noisy image, (c) result by NLM, (d) result by Y-K model, (e) result by PM, (f) result by TV, (g) result by MTV, and (h) result by our model.

regions, $s$ is small, so $\varphi(s) \rightarrow 0$. From the proposed model, we can see that when $\varphi(s) \rightarrow 0$, which means the pixel locates in the edge, TV regularization term is adopted; when $\varphi(s) \rightarrow 1$, which means the pixel belongs to the smooth region, Tikhonov regularization is used. So we can see from our model (8) the following.

(1) In the region containing edges, when $\varphi(s)$ is close to 0 , the new model adopts the total variation (TV) model, so the model is good at preserving the edges of the image.
(2) In the smooth areas of the image, $\varphi(s)$ is close to 1 , and the new model will highlight Tikhonov regularization model, so the model can eliminate the staircase effect.

(3) In the flat areas of image containing less image features and much noise, Tikhonov regularization model and total variation model work together.

3.2. Numerical Implementation. To solve (8), we use split Bregman method proposed by Goldstein and Osher [23]. Split Bregman is a flexible and effective tool for solving 


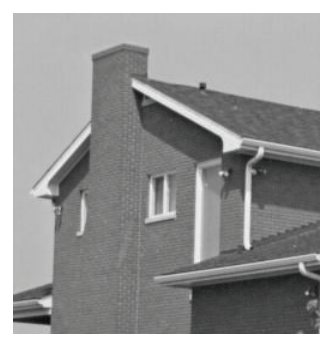

(a)

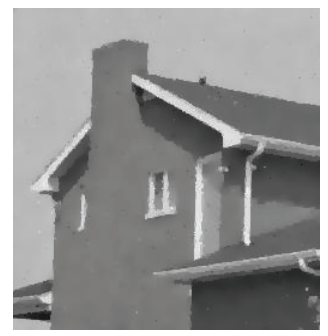

(e)

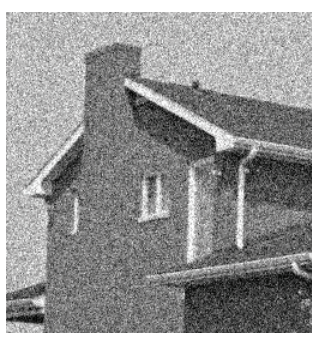

(b)

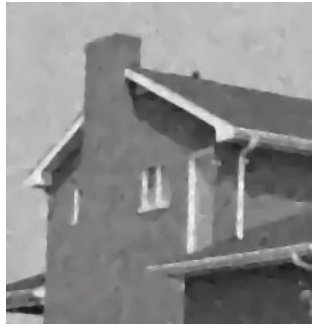

(f)

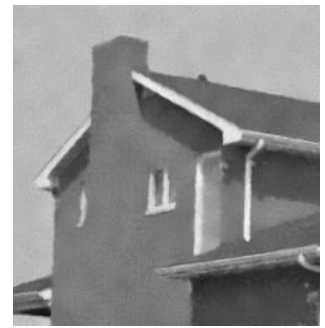

(c)

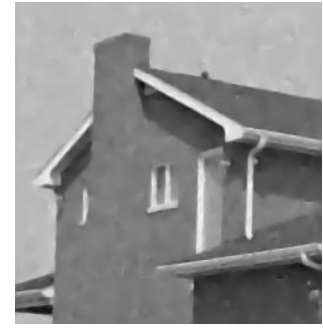

(g)

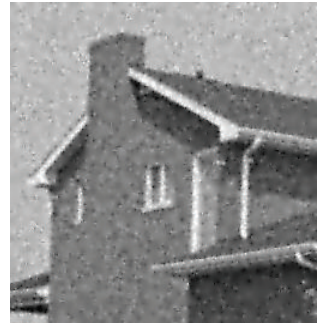

(d)

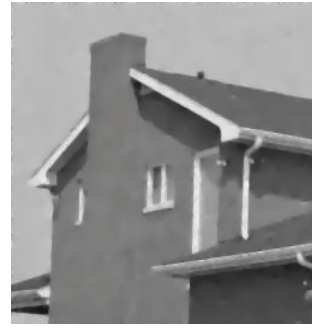

(h)

FIGURE 5: Results of the house image by the five models: (a) original image, (b) noisy image, (c) result by NLM, (d) result by Y-K model, (e) result by PM, (f) result by TV, (g) result by MTV, and (h) result by our model.

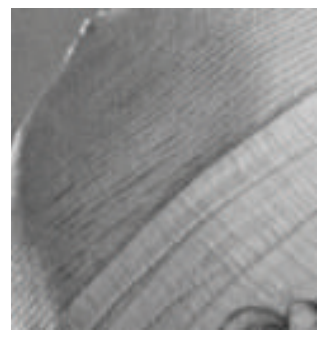

(a)

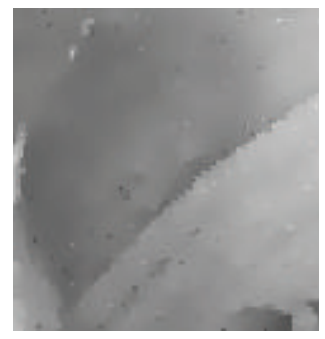

(e)

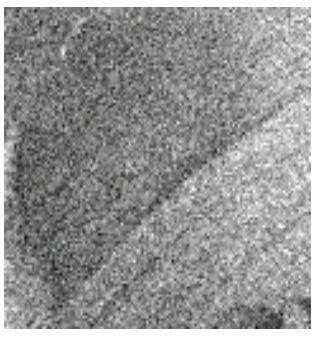

(b)

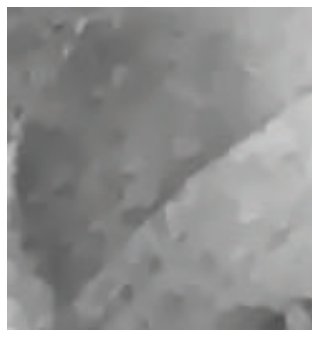

(f)

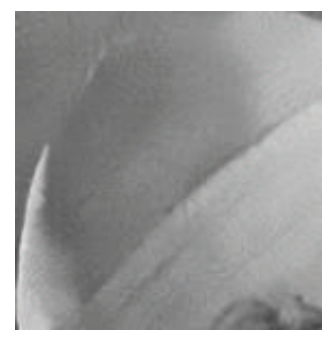

(c)

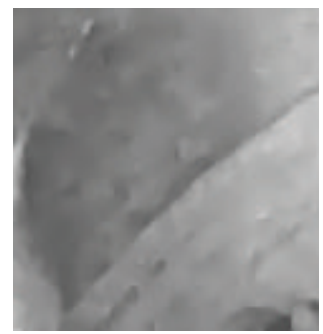

(g)

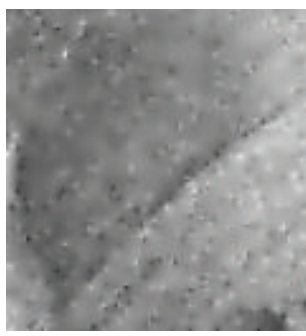

(d)

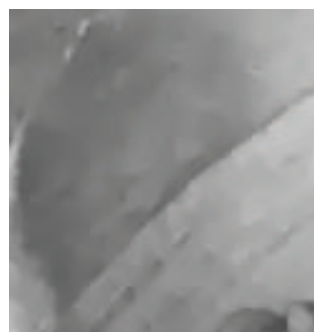

(h)

FIGURE 6: A part of the results of the lena image by the five models: (a) original image, (b) noisy image, (c) result by NLM, (d) result by Y-K model, (e) result by PM, (f) result by TV, (g) result by MTV, and (h) result by our model.

various inverse problems. Split Bregman idea is to use splitting operator and Bregman iteration to solve the constrained minimization problems. First, we convert (8) into the constrained minimization problem by introducing an auxiliary variable $f$, so

$$
\begin{gathered}
\min _{u}\left\{\int \int _ { \Omega } \left[(1-\varphi(s))\|\nabla u\|_{\mathrm{TV}}+\varphi(s)\|\nabla u\|_{2}^{2}\right.\right. \\
\left.\left.+\frac{\lambda}{2}\left(f-u_{0}\right)^{2}\right] d x d y\right\},
\end{gathered}
$$

where $\varphi(s)=\varphi(|\nabla u|) . \varphi$ is used to replace $\varphi(|\nabla u|)$. We can see that the first and second terms are not directly crossed. Second, by introducing the variable $b$, the constrained minimization problem (10) can be converted to the following unconstrained problem:

$$
\begin{gathered}
\min _{u, f, b}\left\{\int \int _ { \Omega } \left[(1-\varphi)\|\nabla u\|_{\mathrm{TV}}+\varphi\|\nabla u\|_{2}^{2}\right.\right. \\
\left.+\frac{\lambda}{2}\left(f-u_{0}\right)^{2}\right] d x d y \\
\left.+\frac{\beta}{2}(u-f+b)^{2}\right\},
\end{gathered}
$$

s.t. $f=u$, 
where the penalty parameter $\beta$ is a position constant. Using split Bregman method, the unconstrained problem (11) can be solved by the following iterative equations:

$$
\begin{gathered}
u^{n+1}=\arg \min _{u}\left\{\iint_{\Omega}\left[(1-\varphi)\|\nabla u\|_{\mathrm{TV}}+\varphi\|\nabla u\|_{2}^{2}\right] d x d y\right. \\
\left.+\frac{\beta}{2}\left(u-f^{n}+b^{n}\right)^{2}\right\}, \\
f^{n+1}=\arg \min _{f}\left\{\frac{\lambda}{2} \iint_{\Omega}\left(f-u_{0}\right)^{2} d x d y\right. \\
\left.+\frac{\beta}{2}\left(u^{n+1}-f+b^{n}\right)^{2}\right\}, \\
b^{n+1}=b^{n}+u^{n+1}-f^{n+1} .
\end{gathered}
$$

To solve the first problem in (12), we let

$$
\begin{aligned}
F\left(u, x, y, u_{x}, u_{y}\right)= & (1-\varphi)\|\nabla u\|_{\mathrm{TV}}+\varphi\|\nabla u\|_{2}^{2} \\
& +\frac{\lambda}{2}\left(f-u_{0}\right)^{2}+\frac{\beta}{2}\left(u-f^{n}+b^{n}\right)^{2} .
\end{aligned}
$$

The partial derivatives of (13) are showed as follows:

$$
\begin{aligned}
& F_{u}=\beta\left(u-f^{n}+b^{n}\right), \\
& F_{u_{x}}=\left(\frac{1-\varphi}{|\nabla u|}+2 \varphi\right) u_{x}, \\
& F_{u_{y}}=\left(\frac{1-\varphi}{|\nabla u|}+2 \varphi\right) u_{y} .
\end{aligned}
$$

Therefore the Euler-Lagrange equation of (14) is

$$
\begin{aligned}
& -F_{u}+\frac{\partial}{\partial x} F_{u_{x}}+\frac{\partial}{\partial x} F_{u_{y}} \\
& =\operatorname{div}\left[(1-\varphi) \frac{\nabla u}{|\nabla u|}+2 \varphi \nabla u\right]-\beta\left(u-f^{n}+b^{n}\right) \\
& =0 .
\end{aligned}
$$

According to the gradient descent method, we can derive

$$
u_{t}^{n+1}=\operatorname{div}\left[(1-\varphi) \frac{\nabla u}{|\nabla u|}+\varphi \nabla u\right]-\beta\left(u-f^{n}+b^{n}\right) .
$$

We also derive the Euler-Lagrange equation of the second problem in (12) as follows:

$$
\lambda\left(f-u_{0}\right)-\beta\left(u^{n+1}-f+b^{n}\right)=0 .
$$

This is a linear equation of $f$, so additional operator split (AOS) iteration is used to solve (17).

In sum, the new model can be implemented as follows.

Algorithm 1. The proposed algorithm for image denoising is as follows.
Step 1. Input the observed image $u_{0}$.

Step 2. Initialization: $u^{0}=f^{0}=u_{0}, b^{0}=0$, and $n=0$.

Step 3. Compute $u^{n+1}$ according to (16).

Step 4. Compute $f^{n+1}$ according to (17).

Step 5. Compute $b^{n+1}$ according to the third term of (12).

Until the stop condition is satisfied.

\section{Experiments}

To evaluate the performance of the proposed model, in this section, we have compared our model with other models in terms of the visual quality of denoised images, peak signal to noise ratio (PSNR), and structural similarity index measure (SSIM). PSNR is defined by

$$
\text { PSNR }=10 \log _{10}\left(\frac{255^{2}}{\mathrm{MSE}}\right)(\mathrm{db}),
$$

with

$$
\operatorname{MSE}=\frac{1}{|\Omega|} \sum \sum\left(u-u_{0}\right)^{2}
$$

where $|\Omega|$ denotes the image size, $u$ is the recovered image, and $u_{0}$ is the noise-free original image. MSE represents the noise variance in the recovered image. PSNR is used to measure the noise removal effect and the larger value indicates the smoother recovered image. However, PSNR sometimes disaccords with human visual judgments. So, SSIM (structural similarity) is used to assess the noise removal quality because the SSIM criterion is closer to the human vision system [24]. SSIM is defined by

$$
\operatorname{SSIM}\left(u, u_{0}\right)=\frac{\left(2 \mu_{u} \mu_{u_{0}}+c 1\right)\left(2 \sigma_{u u_{0}}+c 2\right)}{\left(\mu_{u}^{2}+\mu_{u_{0}}^{2}+c 1\right)\left(\sigma_{u}^{2}+\sigma_{u_{0}}^{2}+c 2\right)}
$$

where $\mu_{u}$ and $\sigma_{u}^{2}$ are the mean and variance of $u$, respectively, $\sigma_{u u_{0}}$ is the covariance of $u$ and $u_{0}$, and $c_{1}$ and $c_{2}$ are two constants to avoid instability. SSIM measures the structure similarity between the original image and the recovered image. A stopping condition for all experiments is defined as follows:

$$
\frac{\left|u^{n+1}-u^{n}\right|}{\left|u^{n}\right|} \leq \varepsilon
$$

Here $\varepsilon$ is a given positive number. We set $\varepsilon=10 e-3$ in the paper. The other denoising parameters in the experiments are set as follows: the time step $\Delta t=0.2$ and grid step size $h=1$, $\lambda=0.02$, and $L=1$ to 25 . We find that $L$ has a deep influence on the denoising results. All experiments were implemented by MATLAB R2009a and performed on 64-bit Windows 7 on the desk with an Intel CPU of $1.7 \mathrm{GHz}$ and $4 \mathrm{~GB}$ memory. 
TABLE 1: PSNR and SSIM comparisons with different methods for the synthetic image $(256 \times 256)$.

\begin{tabular}{lcccccc}
\hline Performance & NLM & Y-K & PM & TV & MTV & Our method \\
\hline PSNR & 26.67 & 25.39 & 29.15 & 29.06 & 31.61 & 32.43 \\
SSIM & 0.71 & 0.65 & 0.93 & 0.92 & 0.95 & 0.96 \\
Time (seconds) & 24.53 & 36.80 & 1.63 & 7.26 & 7.20 & 9.25 \\
\hline
\end{tabular}

TABLE 2: PSNR comparison with different methods for noisy image $(256 \times 256)$.

\begin{tabular}{lccccc}
\hline Method & Lena & Boat & House & Peppers & Cameraman \\
\hline Noisy image & 18.58 & 18.61 & 18.57 & 28.56 & 28.82 \\
NLM & 29.56 & 28.61 & 27.79 & 24.23 & 27.71 \\
Y-K & 27.42 & 25.39 & 26.16 & 28.371 & 23.81 \\
PM & 29.52 & 27.81 & 29.92 & 27.91 & 28.05 \\
TV & 29.34 & 27.18 & 31.37 & 27.79 & 26.22 \\
MTV & 30.76 & 28.43 & 31.94 & 29.32 & 28.27 \\
Our method & 31.55 & 29.23 & & \\
\hline
\end{tabular}

4.1. Experiments with Synthetic Images. Figure 1 shows the comparison of some denoising methods on a $256 \times 256$ synthetic image. Figure 1(a) is the original image and Figure 1(b) is the noisy image with Gaussian noise whose standard deviation is 30 and mean value is zero. Figures $1(\mathrm{c})-1(\mathrm{~h})$ show the denoising results of nonlocal means model [2], Y-K model [11], Perona-Malik model [4], total variation (TV) method [7], modified TV (MTV) [25], and our proposed model. Figure 2 shows the locally enlarged images of Figure 1. From Figures 1 and 2, we can observe that our model yields better results in the visual sense than other models in the visual sense, since it eliminates the staircase effect of TV model and preserves edges as well as TV model. In other words, our model can take advantage of both the filter based on Tikhonov regularization and the one based on total variation regularization.

Table 1 shows the PSNR, SSIM, and computational times of Figure 1. From Table 1, we also see that our model also outperforms other models in PSNR and SSIM. The experiments of our model are solved iteratively like TV, PM, and MTV. Seen from Table 1, the proposed method uses less computational time than Y-K and NLM, but the computational time of our proposed method is higher than those of TV, PM, and MTV.

4.2. Experiments with Natural Images. To verify the performance of our model comprehensively, we have also implemented the quantitative and qualitative evaluation on the natural images from http://decsai.ugr.es/cvg/dbimagenes/. Figures 3, 4, and 5 exhibit the denoising results on the noisy images "lena," "peppers," and "house" with $\sigma=30$. The figures show that the denoising results of our model look better than other models. The results of TV model and modified TV cause the staircase effect. Blurring edges come out in PeronaMalik model. The result of Y-K has speckles. Figure 6 shows the partial enlarged images of Figure 3. Tables 2 and 3 show the PSNR and SSIM of our experiments, from which we can see that the proposed algorithm in all noise power has the highest PSNR and SSIM among the six methods. In addition, we show that the experimental results on the "lena" image with $\sigma=10,20,30,40$, and 50 in Figure 7 and Tables 4 and 5 give the quantitative experimental results of PSNR and SSIM, respectively. These experimental results demonstrate that our model has better performance.

4.3. Comparison with BM3D. Block-matching and 3D filtering (BM3D) [3] is the state-of-the-art denoising method, which enhances the sparsity by grouping similar 2D image blocks into $3 \mathrm{D}$ groups based on the fact that an image has a locally sparse representation in transform-domain. We compare the proposed model with $\mathrm{BM} 3 \mathrm{D}$ whose code is downloaded from http://www.cs.tut.fi/ foi/GCF-BM3D/. As shown in Figure 8 and Table 6, we find that the proposed model is not necessarily better than BM3D in removing the noises from the images. But, our model is still novel considering that it is a significant improvement on the other models based on PDE.

\section{Conclusion}

To eliminate the so-called staircase effect in many known image denoising models and speckles in the high-order PDE, we propose an improved model for image denoising with a weighted combination of Tikhonov regularization and total variation regularization. Numerical experiments show that our proposed model can recover the images from the noisy images, has the highest PSNR and SSIM among the six methods and can preserve important structures, such as edges and corners. 
TABLE 3: SSIM comparison with different methods for noisy image $(256 \times 256)$.

\begin{tabular}{lccccc}
\hline Method & Lena & Boat & House & Peppers & Cameraman \\
\hline Noisy image & 0.22 & 0.29 & 0.23 & 0.29 & 0.81 \\
NLM & 0.76 & 0.71 & 0.82 & 0.59 & 0.80 \\
Y-K & 0.69 & 0.61 & 0.60 & 0.83 & 0.62 \\
PM & 0.80 & 0.72 & 0.82 & 0.81 & 0.81 \\
TV & 0.79 & 0.71 & 0.81 & 0.82 & 0.75 \\
MTV & 0.83 & 0.75 & 0.84 & 0.84 & 0.82 \\
Our method & 0.85 & 0.76 & 0.85 & \\
\hline
\end{tabular}

TABLE 4: PSNR comparison with different methods for the "lena" image.

\begin{tabular}{lccccc}
\hline$\sigma /$ PSNR $_{0}$ & $10 / 28.12$ & $20 / 22.13$ & $30 / 18.53$ & $40 / 16.09$ & 28.01 \\
\hline NLM & 34.95 & 31.65 & 29.56 & 25.2 & 26.82 \\
Y-K & 33.04 & 29.63 & 27.34 & 28.84 & 22.87 \\
PM & 33.94 & 30.91 & 29.42 & 28.1 & 28.34 \\
TV & 33.96 & 31.01 & 30.52 & 29.95 & 27.25 \\
MTV & 34.22 & 31.89 & 31.55 & 30.93 & 29.31 \\
Our method & 34.47 & 32.19 & & 30.15 \\
\hline
\end{tabular}

TABLE 5: SSIM comparison with different methods for the "lena" image.

\begin{tabular}{lccccc}
\hline$\sigma /$ SSIM & $10 / 0.61$ & $20 / 0.35$ & $30 / 0.22$ & $40 / 0.15$ & 0.69 \\
\hline NLM & 0.9 & 0.83 & 0.76 & 0.58 & 0.63 \\
Y-K & 0.86 & 0.77 & 0.69 & 0.78 & 0.45 \\
PM & 0.89 & 0.83 & 0.79 & 0.77 & 0.77 \\
TV & 0.89 & 0.83 & 0.8 & 0.81 & 0.74 \\
MTV & 0.89 & 0.84 & 0.83 & 0.83 & 0.79 \\
Our method & 0.9 & 0.85 & 0.85 & 0.8 \\
\hline
\end{tabular}

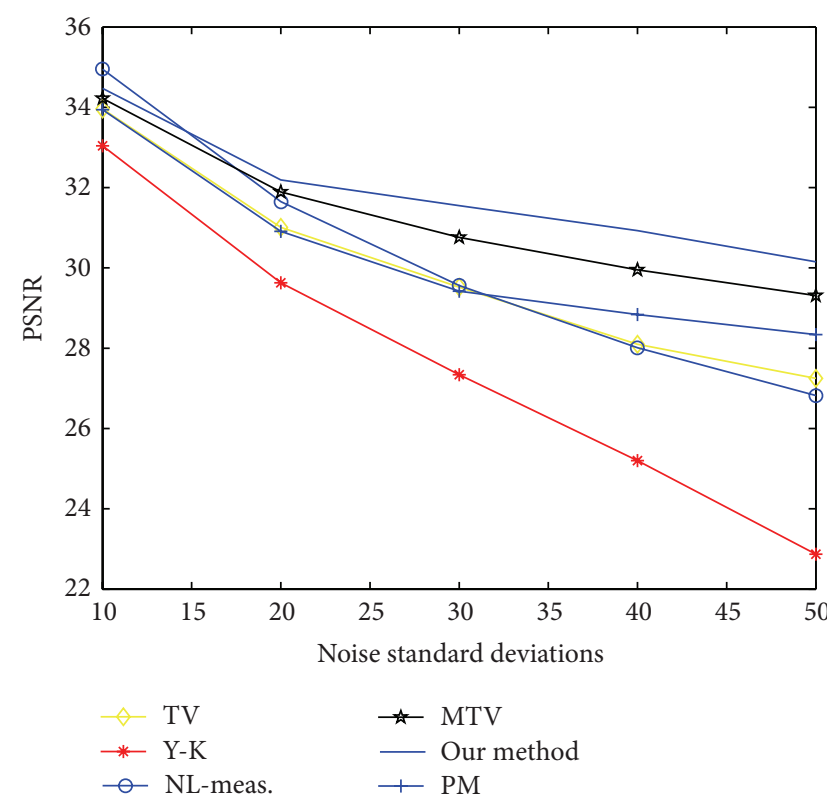

(a) PSNR

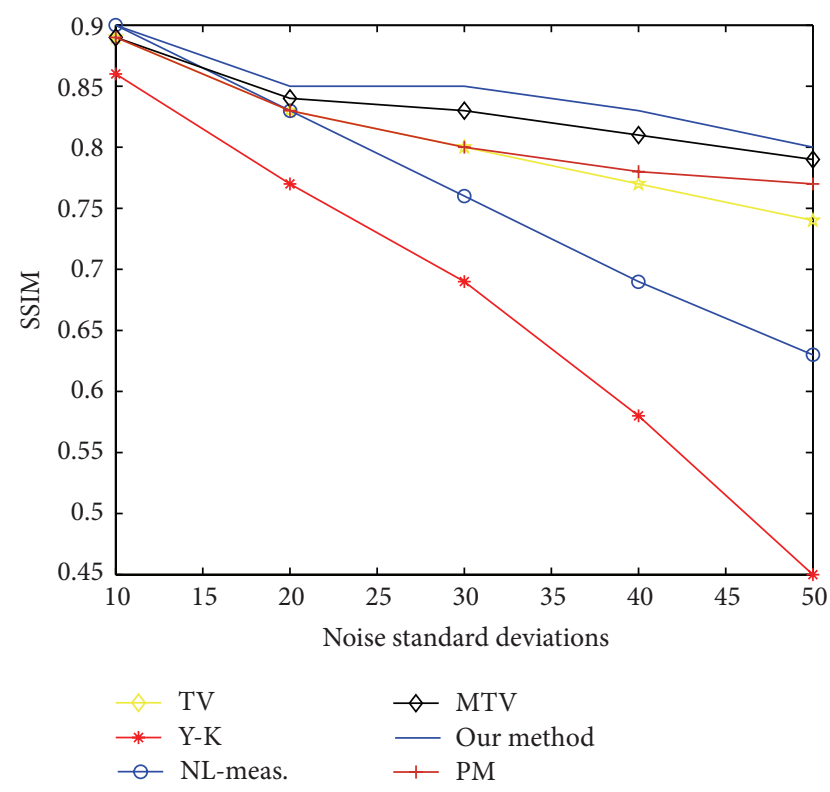

(b) SSIM

FIGURE 7: PSNR and SSIM values of six models for denoising lena image. 

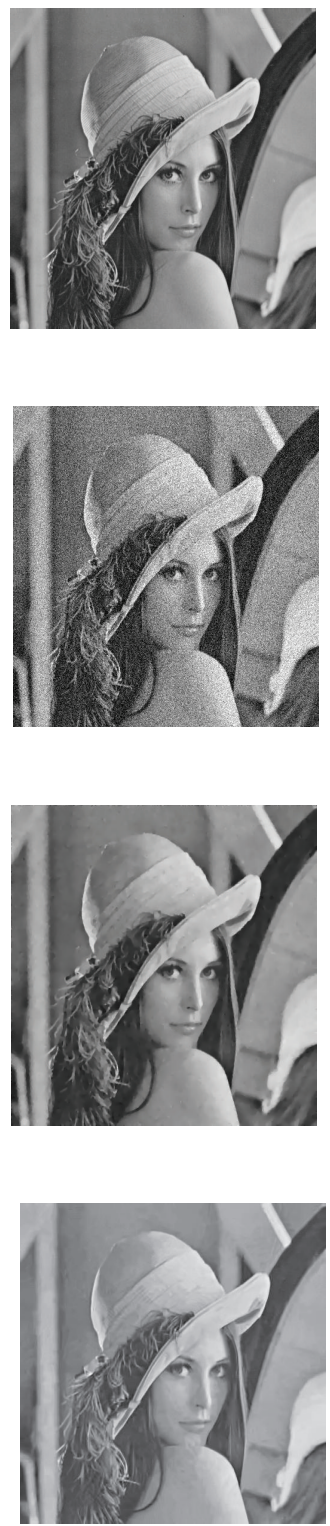

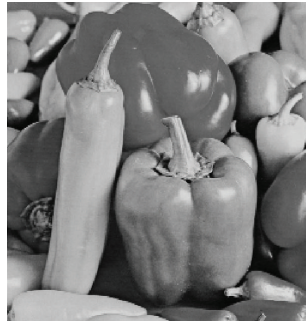

(a)
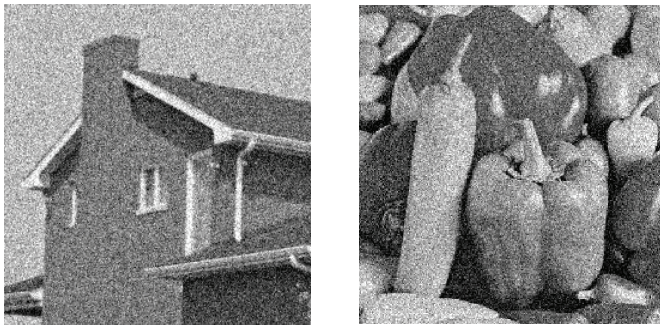

(b)
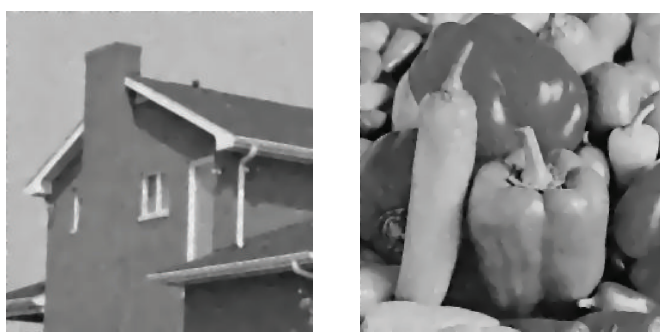

(c)
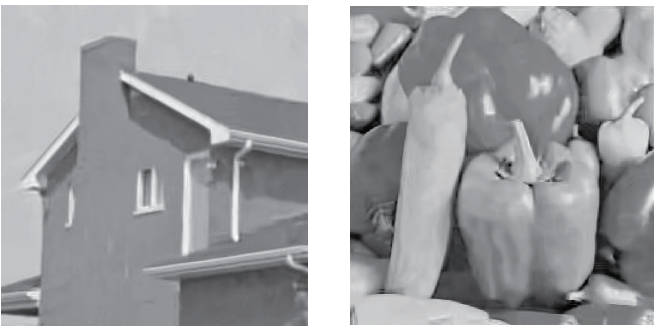

(d)

FIGURE 8: Results of our model and BM3D: (a) original image, (b) noisy image with the mean value of 0 and standard deviation of $\sigma=30$, (c) results by our model, and (d) results by BM3D.

TABLE 6: PSNR and SSIM comparisons between our model and BM3D.

\begin{tabular}{lccc}
\hline Performance (SSIM/PSNR) & Lena & Peppers & House \\
\hline Our model & $0.85 / 31.55$ & $0.84 / 29.32$ & $0.85 / 31.94$ \\
BM3D & $0.86 / 31.46$ & $0.86 / 29.28$ & $0.89 / 32.11$
\end{tabular}

\section{Conflict of Interests}

The authors declare that there is no conflict of interests regarding the publication of this paper.

\section{Acknowledgments}

This work is supported by the National Science Foundation of China (no. 61070227) and NASF-Guangdong Joint Foundation (Key Project) (no. U1135003).

\section{References}

[1] P. Chatterjee and P. Milanfar, "Is denoising dead?" IEEE Transactions on Image Processing, vol. 19, no. 4, pp. 895-911, 2010.

[2] A. Buades, B. Coll, and J. M. Morel, "A review of image denoising algorithms, with a new one," Multiscale Modeling and Simulation, vol. 4, no. 2, pp. 490-530, 2005. 
[3] K. Dabov, A. Foi, V. Katkovnik, and K. Egiazarian, "Image denoising by sparse 3-D transform-domain collaborative filtering," IEEE Transactions on Image Processing, vol. 16, no. 8, pp. 2080-2095, 2007.

[4] P. Perona and J. Malik, "Scale-space and edge detection using anisotropic diffusion," IEEE Transactions on Pattern Analysis and Machine Intelligence, vol. 12, no. 7, pp. 629-639, 1990.

[5] D. Chen, Y. Chen, and D. Xue, "Three fractional-order TVL2 models for image denoising," Journal of Computational Information Systems, vol. 9, no. 12, pp. 4773-4780, 2013.

[6] M. R. Hajiaboli, "An anisotropic fourth-order diffusion filter for image noise removal," International Journal of Computer Vision, vol. 92, no. 2, pp. 177-191, 2011.

[7] L. I. Rudin, S. Osher, and E. Fatemi, "Nonlinear total variation based noise removal algorithms," Physica D: Nonlinear Phenomena, vol. 60, no. 1-4, pp. 259-268, 1992.

[8] P. Blomgren, T. F. Chan, and P. Mulet, "Extensions to total variation denoising," in Advanced Signal Processing: Algorithms, Architectures and Implementations VII, Proceedings of SPIE, pp. 367-375, San Diego, Calif, USA, July 1997.

[9] M. Lysaker, A. Lundervold, and X. C. Tai, "Noise removal using fourth-order partial differential equation with applications to medical magnetic resonance images in space and time," IEEE Transactions on Image Processing, vol. 12, no. 12, pp. 1579-1589, 2003.

[10] A. S. Khare, R. Mohan, and S. Sharma, "An efficient image denoising method based on fourth-order partial differential equations," International Journal of Advanced Computer Research, vol. 3, no. 9, pp. 126-131, 2013.

[11] Y.-L. You and M. Kaveh, "Fourth-order partial differential equations for noise removal," IEEE Transactions on Image Processing, vol. 9, no. 10, pp. 1723-1730, 2000.

[12] S. Zheng, Z. Pan, G. Wang, and X. Yan, "A variational model of image restoration based on first and second order derivatives and its split Bregman algorithm," in Proceedings of the $3 \mathrm{rd}$ IEEE/IET International Conference on Audio, Language and Image Processing (ICALIP '12), pp. 860-865, Shanghai, China, July 2012.

[13] S. Oh, H. Woo, S. Yun, and M. Kang, "Non-convex hybrid total variation for image denoising," Journal of Visual Communication and Image Representation, vol. 24, no. 3, pp. 332-344, 2013.

[14] A. Gholami and S. M. Hosseini, "A balanced combination of Tikhonov and total variation regularizations for reconstruction of piecewise-smooth signals," Signal Processing, vol. 93, no. 7, pp. 1945-1960, 2013.

[15] W. Stefan, R. A. Renaut, and A. Gelb, "Improved total variationtype regularization using higher order edge detectors," SIAM Journal on Imaging Sciences, vol. 3, no. 2, pp. 232-251, 2010.

[16] R. W. Liu, L. Shi, W. Huang, J. Xu, S. C. H. Yu, and D. Wang, "Generalized total variation-based MRI Rician denoising model with spatially adaptive regularization parameters," Magnetic Resonance Imaging, vol. 32, no. 6, pp. 702-720, 2014.

[17] E. J. Candès and F. Guo, "New multiscale transforms, minimum total variation synthesis: applications to edge-preserving image reconstruction," Signal Processing, vol. 82, no. 11, pp. 1519-1543, 2002.

[18] A. Gholami and H. R. Siahkoohi, "Regularization of linear and non-linear geophysical ill-posed problems with joint sparsity constraints," Geophysical Journal International, vol. 180, no. 2, pp. 871-882, 2010.
[19] A. N. Tikhonov, A. S. Leonov, and A. G. Yagola, "Nonlinear illposed problems," in World Congress of Nonlinear Analysts '92, Vol. I-IV (Tampa, FL, 1992), pp. 505-511, de Gruyter, Berlin, Germany, 1996.

[20] C. Pschl, Tikhonov regularization with general residual term [Ph.D. thesis], Leopold-Franzens-Universität, Innsbruck, Austria, 2008.

[21] S. Esedoǵlu and S. J. Osher, "Decomposition of images by the anisotropic Rudin-Osher-Fatemi model," Communications on Pure and Applied Mathematics, vol. 57, no. 12, pp. 1609-1626, 2004.

[22] J. S. Moll, “The anisotropic total variation flow," Mathematische Annalen, vol. 332, no. 1, pp. 177-218, 2005.

[23] T. Goldstein and S. Osher, "The split Bregman method for L1regularized problems," SIAM Journal on Imaging Sciences, vol. 2, no. 2, pp. 323-343, 2009.

[24] Z. Wang, A. C. Bovik, H. R. Sheikh, and E. P. Simoncelli, "Image quality assessment: from error visibility to structural similarity," IEEE Transactions on Image Processing, vol. 13, no. 4, pp. 600612, 2004.

[25] Y. Wang, W. Chen, S. Zhou, T. Yu, and Y. Zhang, "MTV: modified total variation model for image noise removal," Electronics Letters, vol. 47, no. 10, pp. 592-594, 2011. 

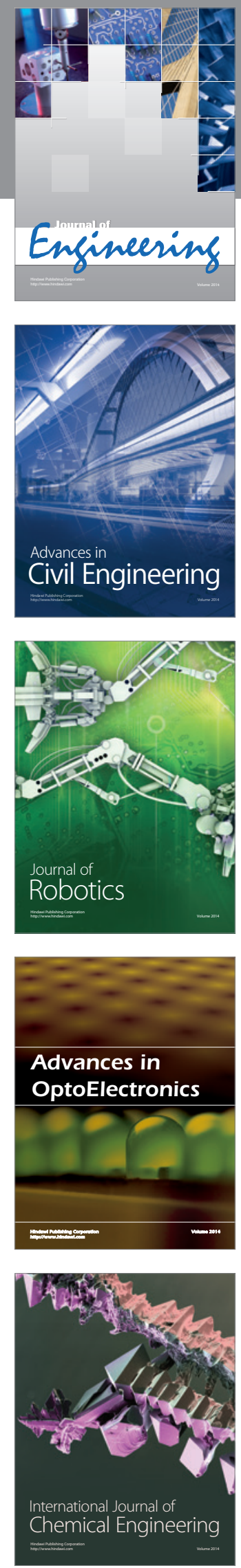

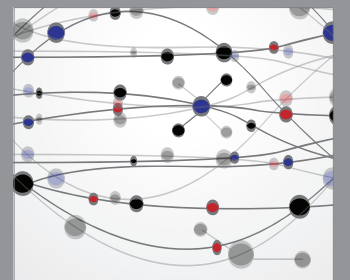

The Scientific World Journal
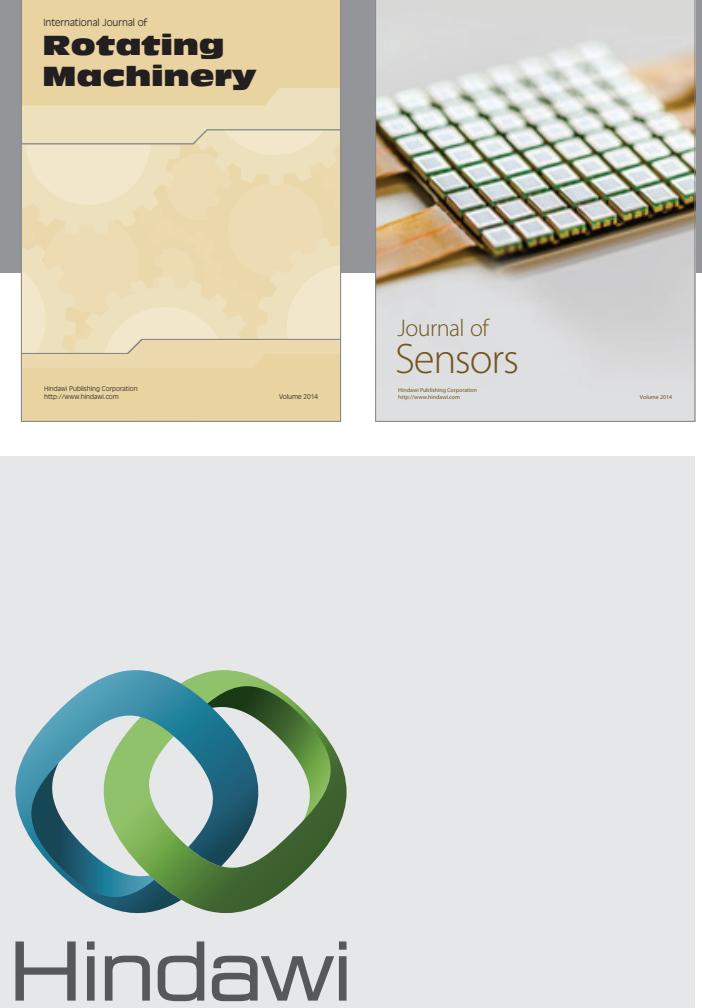

Submit your manuscripts at http://www.hindawi.com
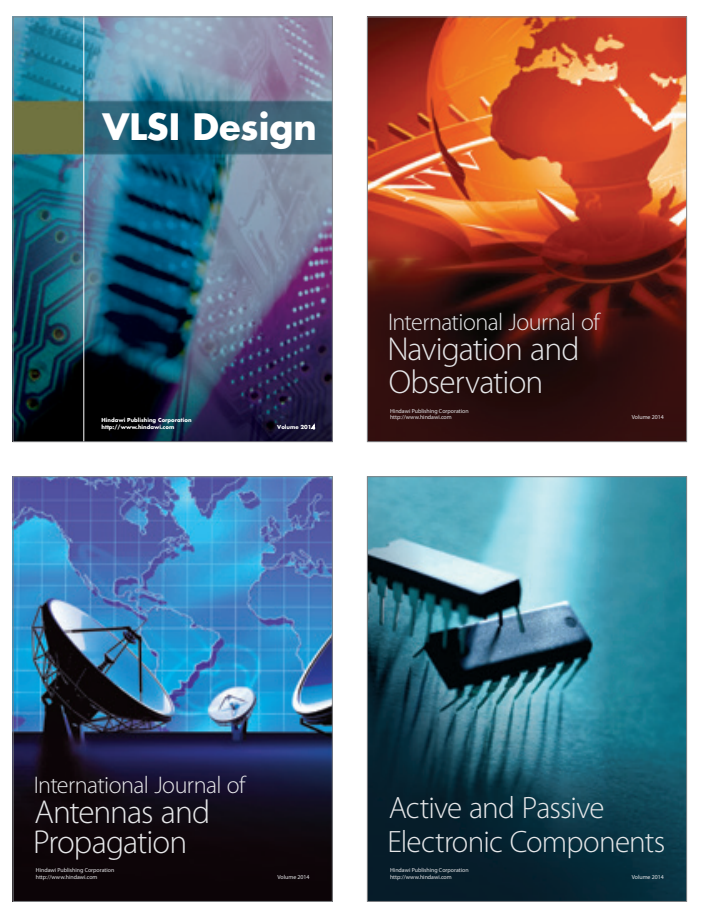
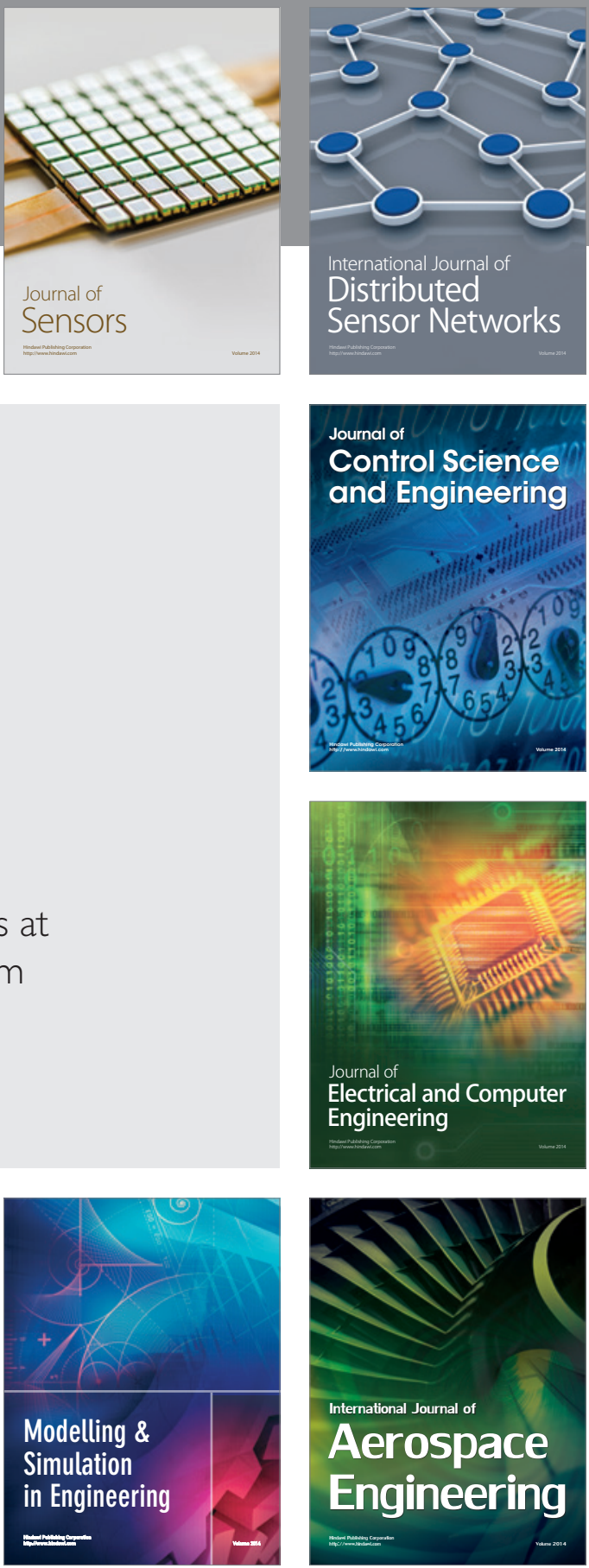

Journal of

Control Science

and Engineering
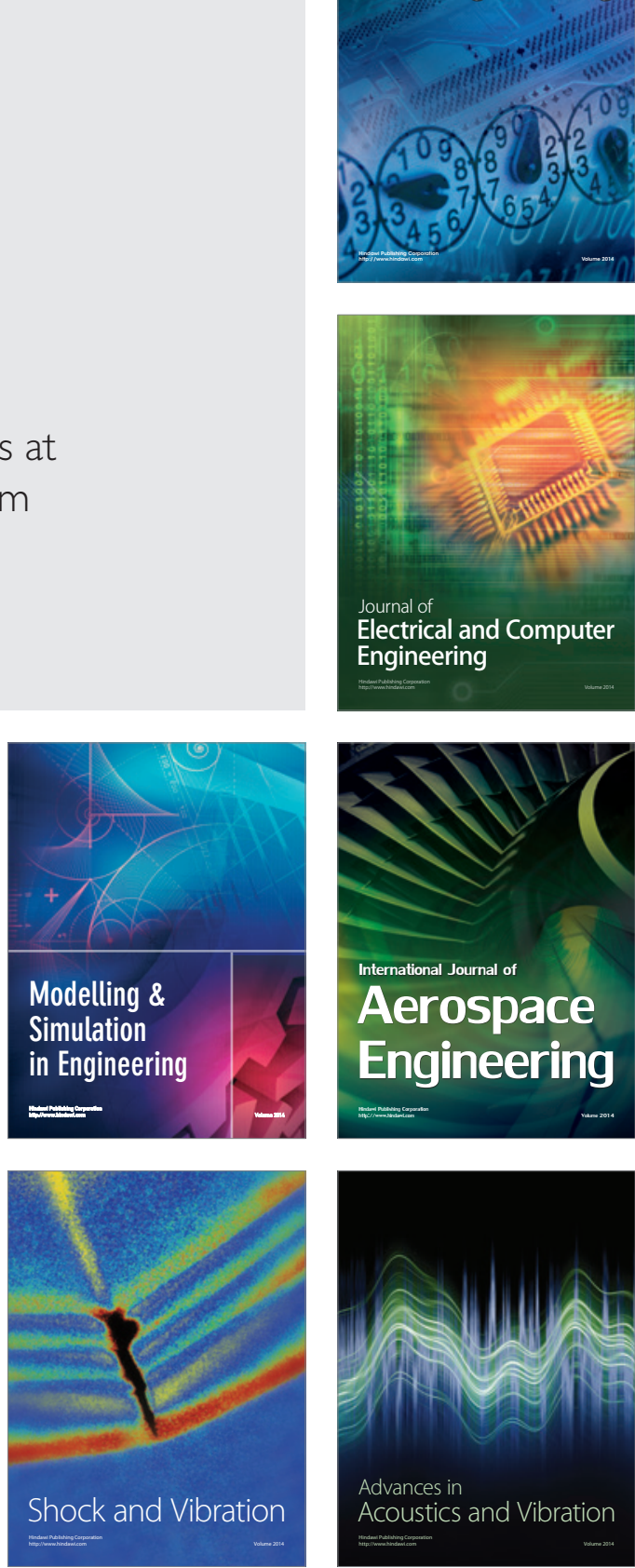